\section{Clinical advantages of using unflattened 6-MV and I0-MV photon beams generated by the medical accelerator Elekta Versa HD based on their dosimetric parameters in comparison to conventional beams}

\begin{abstract}
Photon beams with wide energy ranges from 4 MV to $25 \mathrm{MV}$ are commonly used in radiotherapy nowadays. In recent years, there has been a strong interest in a certain modification of a radiotherapeutic apparatus by the application of the so-called flattening filter-free (FFF) beam. Several advantages of FFF beams over standard flattening filter (FF) beams are noticed, and this technical solution has aroused great interest among radiotherapeutic facilities. The goal of the present study is to investigate the differences between the conventional FF and unflattened FFF 6-MV and 10-MV photon beams in some basic dosimetric parameters and their influence on the whole radiotherapeutic treatment. The data provided here include the detailed characteristics as follows: percent depth dose (PDD), beam profile, edge of a half-profile, total scatter correction factor (TSCF) and head scatter correction factor (HSCF) for FF and FFF 6-MV and 10-MV photon beams from the Elekta Versa HD accelerator in the Katowice Oncology Center in Poland.
\end{abstract}

Keywords: clinical application of FFF beams $\bullet$ dosimetric parameters $\bullet$ unflattened photon beams

\author{
Błażej Baic ${ }^{\bowtie}$ \\ Katowice Oncology Center \\ 26 Raciborska St., 41-074 Katowice, Poland \\ and Institute of Physics \\ University of Silesia \\ 75 Pulku Piechoty 1A St., 41-500 Chorzow, Poland \\ E-mail: blazejbaic@gmail.com \\ R. Kwiatkowski, M. Dybek \\ Katowice Oncology Center \\ 26 Raciborska St., 41-074 Katowice, Poland \\ B. Kozłowska \\ Institute of Physics \\ University of Silesia \\ 75 Pulku Piechoty 1A St., 41-500 Chorzow, Poland
}

Received: 10 October 2018

Accepted: 6 March 2019

\section{Introduction}

Photon beams conventionally used in radiotherapy are shaped by the MultiLeaf Collimator (MLC) and the flattening filter (FF). In recent years, there has been increasing interest in the removal of flattening filter as a factor which gives rise to additional scattering. This kind of beam is called a flattening filter-free (FFF) photon beam or an unflattened beam.

The removal of the filter to obtain FFF beams changes the natural peak shape of the beam profile as compared to the conventional FF photon beam. Given the development of computational techniques in this field, more sophisticated treatment planning systems and treatment techniques, the homogeneous dose distribution is not required. In view of the fact that the dose rate is much higher in FFF beams than in FF beams, the use of FFF beams has increased substantially. Consequently, the exposure time may be shorter and therefore the probability of patient movements between fractions may be reduced.

As stated above, the use of FFF beams is increasingly common in radiotherapy nowadays, meaning that their dosimetric parameters should be well known to radiotherapy staff. A good understanding of the differences between conventional and unflattened photon beams is needed; nonetheless, most of the available publications do not compare conventional FF and FFF photon beams, but essentially focus on their usability. Only a few authors of the publications on FFF beams made a comparison 
between these two kinds of photon beams $[1,2]$. Cashmore described only 6-MV FF and FFF photon beams and their dosimetric parameters such as percent depth dose (PDD), beam profiles, total scatter correction factor (TSCF), head scatter correction factor (HSCF) and wedge factors. All these factors were measured at the open and wedge beams using semiflex detectors for a few field sizes and different depths. Kragl et al. described the beam profiles, PDD, output factors (OFs), leaf transmission and leakage and the surface dose for the 6-MV and 10-MV FF and FFF beams. It should be noted, however, that neither of these authors took into account the clinical applications of FFF beams.

The other available publications on the subject can be divided according to the type of applied accelerators: Elekta Co. [3-5] and Varian Co. [5-10]. All these authors, except Riis and Fogliata, presented dosimetric parameters such as PDD, beam profiles and output factors for FFF beams. Riis et al. depicted only the OF from Elekta Versa HD. Narayanasamy et al. described the wedge factors, MLC characterization and mechanical tests for FFF photon beams and for electron beams. Paynter et al. exhibited head leakage and compared conventional and unflattened beams using both the Elekta Versa HD and Varian True Beam accelerators. Fogliata et al. focused on the quality assurance of FFF beams in terms of field region, field size, penumbra, slope and unflatness for 6-MV and 10-MV FFF beams from Varian True Beam. In addition to the main features, some authors working on Varian accelerators presented a few different parameters. Mohammed et al. described the surface dose and dose rate of 6-MV beams, Pichandi et al. introduced the penumbra of 6-MV and 10-MV FFF beams. Ting et al. presented the dynamic leaf gap for two kinds of beams. Vassiliev et al. described the dose rate data and MLC transmission factors of 6-MV and 18-MV beams without flattening filter.

Moreover, particular mention should be made of a few available conference presentations introducing various radiotherapeutic centres interested in FFF beams and testing them. The authors presented their results at industrial conferences, and their achievements are accessible on the Internet, but were not published in scientific journals [11-13]. For instance, Duane et al. presented the basic information on the FFF 6-MV photon beams generated by the tomotherapy accelerator in the National Physical Laboratory. They explored the impact of filter removal on the energy spectrum and ion recombination and their influence on the perturbation effects in small fields. Kry from the University of Texas provided basic information on the parameters of the Varian accelerator generating unflattened beams, such as PDDs, profiles, OF, penumbra and MLC leakage. In addition, he presented dosimetric parameters based on clinical studies and the practical applications of unflattened beams (e.g. prostate intensity-modulated radiotherapy (IMRT) and stereotactic radiotherapy (SRT)). It should be mentioned that none of these publications considered all the possible explorations, characteristics and applications of both photon beams.
The most recent publication on interested photon beams is the Institute of Physics and Engineering in Medicine - IPEM local report [14], which is in essence a guidance for medical physicists on implementing FFF beams in radiotherapy. Budgell et al. described the parameters of unflattened beams such as PDDs, beam profiles and output factors generated by the Elekta and Varian accelerators and compared them with the respective parameters of conventional beams. Moreover, the authors expressed their views on radiation protection which is analysed using parameters such as patient scatter, primary beam, leakage, production of neutrons and possibilities of the clinical use of FFF beams. In the report, the authors also took note of the dosimetric parameters of the beams and their verification using various kinds of detectors. They provided an example of the clinical use of stereotactic radiosurgery (SRS)/body stereotactic radiation therapy (SBRT), intensity-modulated radiotherapy (IMRT)/volumetric modulated arc therapy (VMAT) planning and peripheral doses.

The goal of the present study is to compare some basic dosimetric and physical parameters of the most commonly used FF and FFF 6-MV and 10-MV photon beams. This publication presents the advantages of FFF on the basis of the measured differences between two kinds of photon beams taking into account the patient's comfort during the treatment. The dosimetric data contain the following characteristics: PDD, beam profile, edge of a half-profile, head scatter correction factor (HSCF) and total scatter correction factor (TSCF). The physical parameters include dose distributions, histograms, treatment time and, in general, the patient's health and well-being. The measurements were performed on the Elekta Versa HD accelerator in the Katowice Oncology Center. The applications of FFF beams in the clinical treatment are presented. Some advantages of beams without flattening filter such as less scattered radiation and easier and more accurate modelling of dose distributions in the treatment planning system are also discussed.

\section{Methods}

All the mentioned parameters, such as profiles, shape of the edge of half-profile, PDD, OF, studied by several different authors and presented in available publications, show the main differences between $\mathrm{FF}$ and FFF beams. The beam profile characterizes a dose distributed along the axes perpendicular to the beam axis. PDD denotes a percentage depth dose. It is a ratio of the absorbed dose at a certain depth in the medium to the dose at the depth, where it reaches its maximum. PDD is visualized as a plot in terms of a percentage of the maximum dose. Head scatter correction factor (HSCF) describes the scattering from the accelerator's head, while total scatter correction factor (TSCF) denotes the complete scattering from the accelerator. Another feature that can be compared in FF and FFF beams is $\mathrm{TPR}_{20,10}$. The tissue phantom ratio (TPR) is the ratio of the dose at the isocenter in water at two 
Table 1. Parameters of FF and FFF photon beams from the Elekta Versa HD accelerator [19]

\begin{tabular}{lcccc}
\hline \multicolumn{1}{c}{ Parameter } & 6-MV FF & 6-MV FFF & 10-MV FF & 10-MV FFF \\
\hline Maximum dose depth $\left(d_{\max }\right)[\mathrm{cm}]$ & 1.5 & 1.7 & 2.1 & 2.4 \\
Percent dose at the depth of $10 \mathrm{~cm}\left(D_{10}\right)$ & 67.5 & 67.5 & 73.0 & 73.0 \\
Quality index $(\mathrm{QI}), \mathrm{TPR} \mathrm{T}_{20,10}$ & 0.68 & 0.68 & 0.73 & 0.72 \\
Minimum nominal value of dose rate $[\mathrm{MU} / \mathrm{min}]$ & 30 & 200 & 30 & 400 \\
Maximum nominal value of dose rate $[\mathrm{MU} / \mathrm{min}]$ & 500 & 1400 & 500 & 2200 \\
\hline
\end{tabular}

different depths, for instance, it is a quotient of the dose at the depth of $20 \mathrm{~cm}$ to the dose at the depth of $10 \mathrm{~cm}\left(D_{20,10}\right)$ [15-17]. When one uses $\mathrm{TPR}_{20,10}$ as a quality index (QI), it is measured by described $D_{20,10}$ and expressed by the Followill equation [18]: $\mathrm{TPR}_{20,10}=1.2661 \cdot D_{20,10}-0.0595$.

The physical parameters characterizing the Elekta Versa HD accelerator used in the present study are summarized in Table 1 . The first three lines describe the depth of the maximum dose $\left(d_{\max }\right)$, the percent dose at the depth of $10 \mathrm{~cm}\left(D_{10}\right)$ and the quality index $\mathrm{TPR}_{20,10}$ for two types of beams, FF and FFF, and for two energies, $6 \mathrm{MV}$ and $10 \mathrm{MV}$. The values of $d_{\max }, D_{10}$ and QI are not significantly different for both beams at the same acceleration voltage. The lack of flattening filter does not affect the quality index which is the same at one voltage for both beams. The last two lines indicate that the minimum and maximum values of dose rates for unflattened FFF photon beams are much higher compared to conventional beams. This feature is very desirable in clinical applications.

\section{Results}

All measurements were carried out in the water phantom of the volume of $40 \times 40 \times 40 \mathrm{~cm}^{3}$ (Blue Phantom - IBA) for 6-MV and 10-MV FF and FFF beams. Each dosimetric parameter was measured in individual way and by specific detector. PDDs were measured by Markus type PPC05 IBA plane-parallel chamber, but others (beam profiles, edge of half-profile and OF) were measured by cylindrical CC13 IBA chamber with the active volume equal to $0.125 \mathrm{~cm}^{3}$.

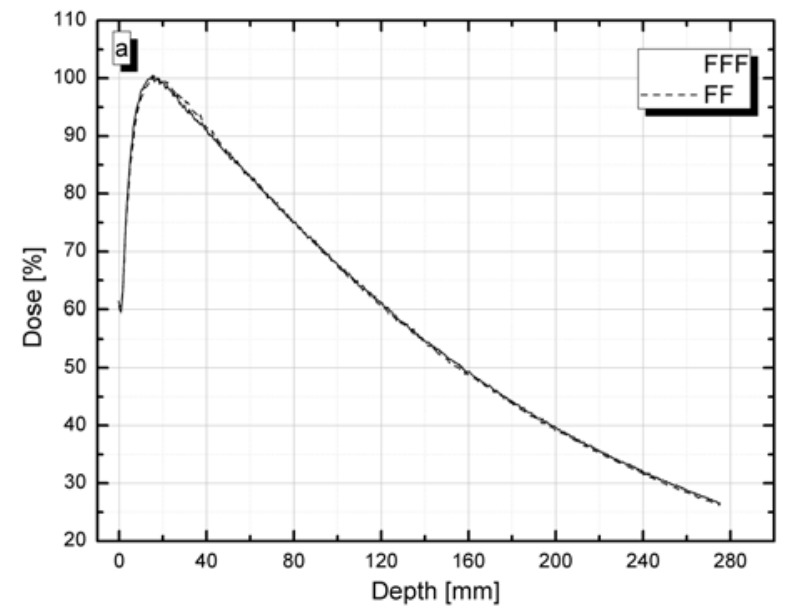

Percent depth dose (PDD) and TPR 20,10

PDD curves were measured starting at the depth equal to $275 \mathrm{~mm}$ up to the water surface $(0 \mathrm{~mm})$. The examples of curves are illustrated in Figs. 1a and $1 \mathrm{~b}$. It can be seen that for the same field size, PDD curves do not differ from each other in both shape and values corresponding to the dose at a given depth.

Table 2 summarizes a comparison of the characteristic values in the quality index for both types of radiation beams generated by the voltage $6 \mathrm{MV}$ and $10 \mathrm{MV}$, respectively. The results show a very good fit of FFF beams in the Elekta Versa HD due to the sophisticated digital technology used to control energy radiation. More importantly, as can be seen in Table 2, the application of FFF beams does not have any influence on the beam quality $\left(\mathrm{TPR}_{20,10}\right)$, so FFF can be commonly used as a conventional photon beam. The difference of $\mathrm{TPR}_{20,10}$ for $10-\mathrm{MV}$ photons is not relevant to the beam quality, but it reflects different values of $D_{10}$ and $D_{20}$.

Table 2. Comparison of measured values of $D_{10}$ and $D_{20}$ for flattening filter (FF) and flattening filter-free (FFF) photon beams from Elekta Versa HD in the Katowice Oncology Center

\begin{tabular}{lrrr}
\hline & $D_{10}[\%]$ & $D_{20}[\%]$ & $\mathrm{TPR}_{20,10} *$ \\
\hline 6-MV FF & 67.81 & 39.61 & 0.68 \\
6-MV FFF & 67.48 & 39.24 & 0.68 \\
Relative difference & \pm 00.33 & \pm 00.37 & \pm 0.00 \\
10-MV FF & 72.45 & 45.49 & 0.74 \\
10-MV FFF & 72.57 & 44.77 & 0.72 \\
Relative difference & \pm 00.12 & \pm 00.72 & \pm 0.02 \\
\hline
\end{tabular}

* $\mathrm{TPR}_{20,10}=1.2661 \cdot D_{20,10}-0.0595$ (Followill et al. [18]).

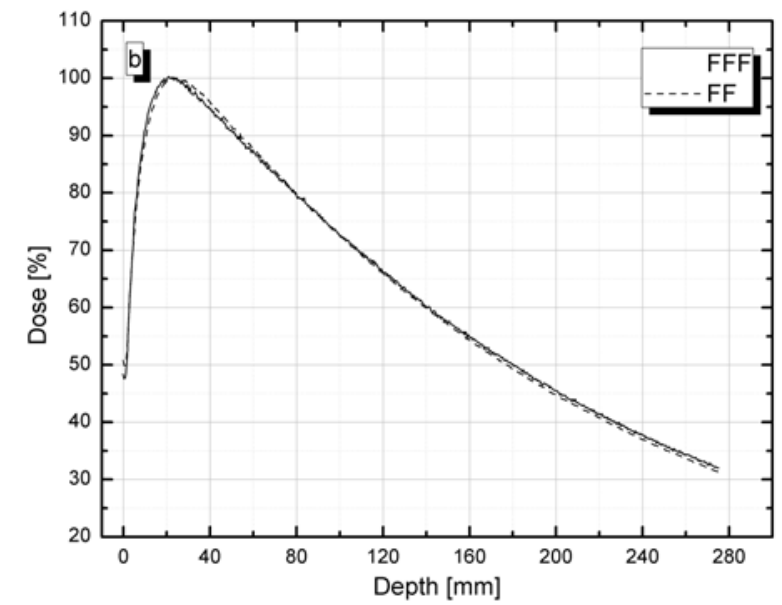

Fig. 1. Percent depth dose curve of FF and FFF (a) 6-MV and (b) 10-MV photon beams for $10 \times 10-\mathrm{cm}^{2}$ field. 

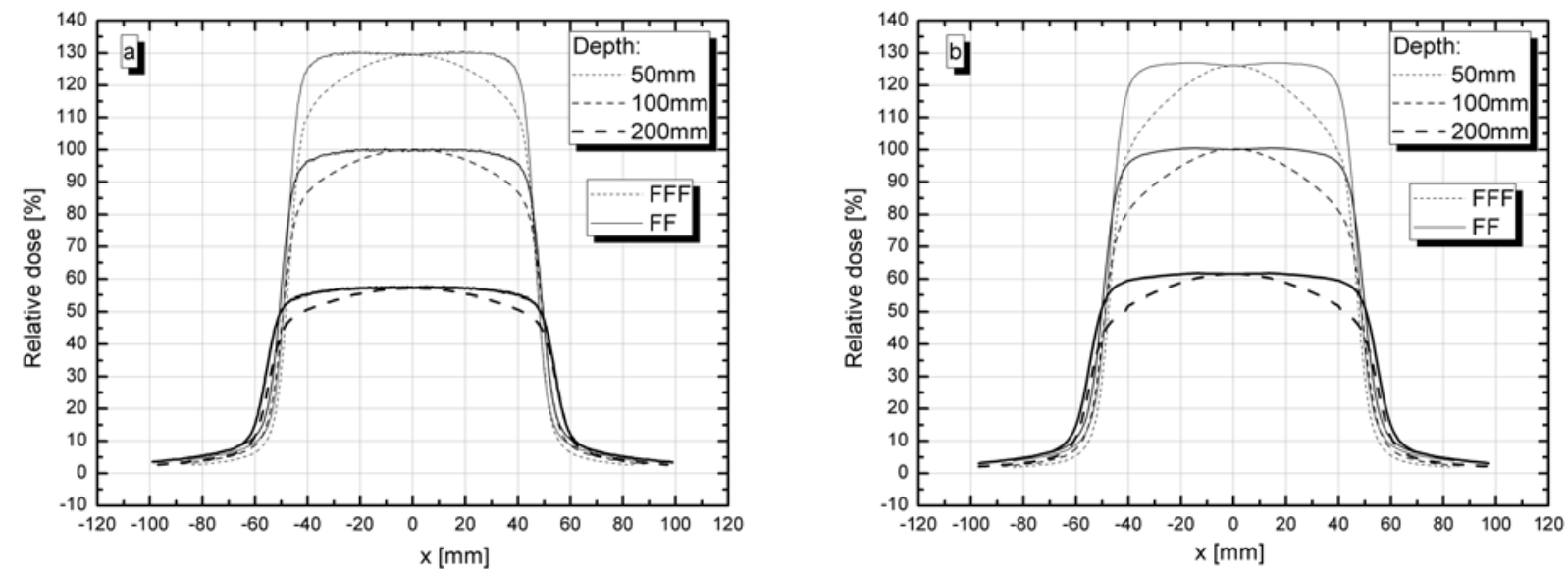

Fig. 2. Differential crossline profile for the flattening filter (FF) and flattening filter-free (FFF) (a) 6-MV and (b) 10 -MV photon beams, field $S=10 \times 10 \mathrm{~cm}^{2}$ for different depth values.

Table 3. Values of relative doses for FF and FFF photon beams in the area outside the central axis of the beam

\begin{tabular}{lcccccc}
\hline \multicolumn{3}{c}{ Relative dose [\%] } \\
\hline Depth [mm] & 50 & 50 & 100 & 100 & 200 & 200 \\
Distance from the axis of the beam [mm] & 20 & 40 & 20 & 40 & 20 & 40 \\
6-MV FF & 130.2 & 125.3 & 99.80 & 96.40 & 57.10 & 55.20 \\
6-MV FFF & 125.2 & 110.2 & 96.95 & 86.82 & 55.41 & 50.69 \\
Relative difference & 5.0 & 15.1 & 2.84 & 9.58 & 1.69 & 4.51 \\
10-MV FF & 126.86 & 119.14 & 100.29 & 95.83 & 61.48 & 59.48 \\
10-MV FFF & 118.87 & 99.03 & 94.90 & 81.17 & 58.83 & 51.90 \\
Relative difference & 7.99 & 20.11 & 6.0 & 14.66 & 2.65 & 7.58 \\
\hline
\end{tabular}

\section{Beam profile characteristics}

Measurements of the percentage dose distributions along the axis perpendicular to the central axis of the radiation beam were also performed for selected fields of regular dimension side from $5 \times 5 \mathrm{~cm}^{2}$ to $30 \times 30 \mathrm{~cm}^{2}$ and to the depths of 50,100 and $200 \mathrm{~mm}$. The examples of curves obtained for a $10 \times 10-\mathrm{cm}^{2}$ field size are illustrated in Figs. $2 \mathrm{~b}$ and $2 \mathrm{~b}$. The biggest difference in the shape of the profile can be reported in the high- and low-dose gradient (the therapeutic area of the radiation beam), in particular for small depths. These differences are more pronounced for the 10-MV than 6-MV photon beams. As the depth increases, the difference in the dose distribution for a certain field size decreases. This phenomenon is most likely associated with a reduction in the amount of low-energy photons with increasing depth in the area of the central axis of the FFF photon beam.

Table 3 summarizes the percentage difference in the relative dose for 6-MV and 10-MV FF and FFF photon beams measured in the area of the central axis outside the beam (high-dose and small-dose gradient). All measurements were taken for three depths $(50,100$ and $200 \mathrm{~mm})$ in the two distances from the beam axis $(20$ and $40 \mathrm{~mm})$. These values were selected on account of the clarity of the relative differences between used beams. In general, when the depth and distance from the beam axis increase, the relative dose goes down. The relative difference is the dependence between the relative dose of the FF beam and FFF beam, with the distance from the beam axis, the depth and the accelerating voltage remaining the same. For all variables, the relative dose is higher for FF beams. This is due to the additional scattering caused by FF. In addition, one can see that for 10-MV beams, the relative differences have higher values. This can be explained by the fact that the FF absorbs more low-energy photons for the high-energy beam.

\section{Edge of half-profile}

The parameter one should pay close attention while analysing these profiles is the change of the relative dose value depending on the depth dimension for $80 \%$ of the field size. The relative dose value is commonly measured for the field size of $10 \times 10 \mathrm{~cm}^{2}$ and always on source skin distance, $\mathrm{SSD}=100 \mathrm{~cm}$, proportionally for different depths. It can be seen in Figs. 3a-3d that for 6-MV and 10-MV FF photon beams, the relative doses vary with the depth, whereas it is not visually observed for FFF. This effect is more pronounced for the 10-MV than 6-MV photon beams. The purpose of the presented changes (Figs. 3a-3d) in the profiles at the edges of the irradiation field is to check the shape disparities of the profiles varying with the depth of the FF and FFF 6-MV and 10-MV photon beams.

The numerical values of the observed effects are summarized in Table 4 , where the relative doses for $80 \%$ of the field size for four depths for all the studied photon beams are presented. For FF beams, the values of the relative doses are bigger than the 

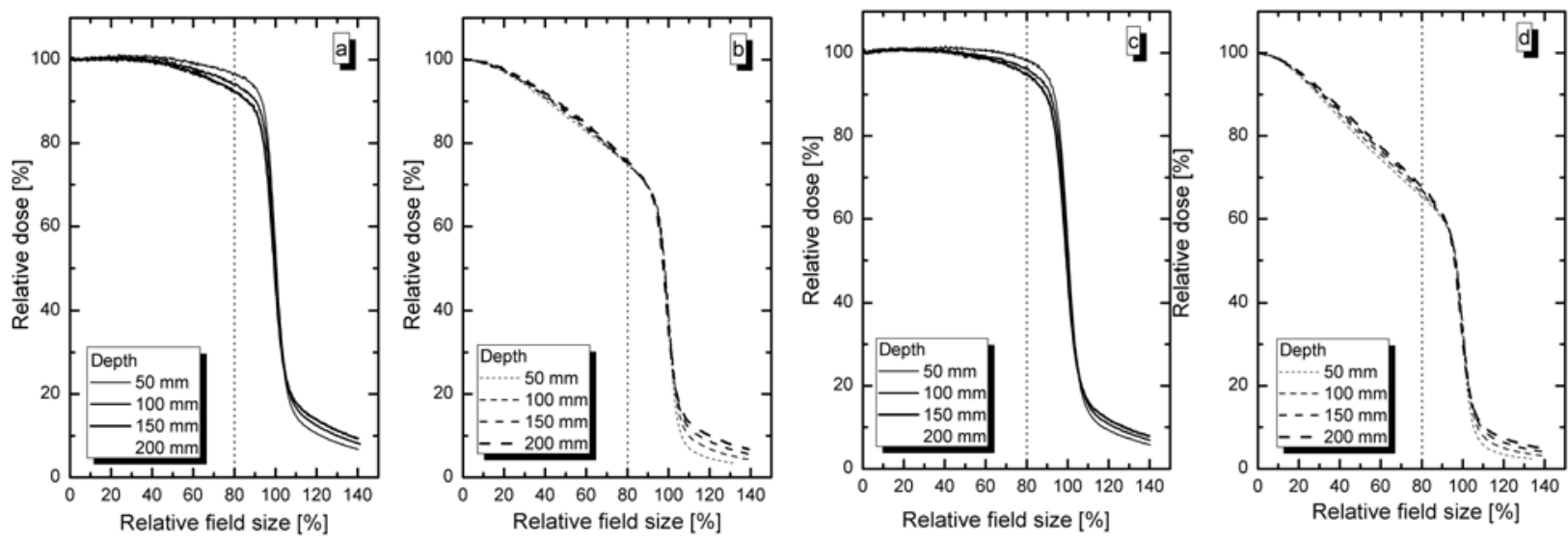

Fig. 3. Edge of lateral half-profiles for (a) 6-MV FF, (b) 6-MV FFF, (c) 10-MV FF and (d) 10-MV FFF photon beams at different depths for the field size of $20 \times 20 \mathrm{~cm}^{2}$. Profiles have been standardized to $100 \%$ on the central axis.

Table 4. Values of relative doses for a relative field size equal to $80 \%$ for FF and FFF photon beams for different depths

\begin{tabular}{ccrrr}
$\begin{array}{c}\text { Depth } \\
{[\mathrm{mm}]}\end{array}$ & \multicolumn{4}{c}{$\begin{array}{c}\text { Relative dose } \\
{[\%]}\end{array}$} \\
\hline 50 & 99.32 & 74.98 & 100.76 & 65.31 \\
100 & 96.68 & 75.05 & 98.29 & 66.25 \\
150 & 94.09 & 75.22 & 96.31 & 66.99 \\
200 & 92.37 & 75.72 & 94.83 & 67.87 \\
\hline
\end{tabular}

corresponding ones for FFF beams. This is due to the fact that all the profiles are normalized to $100 \%$ of the relative dose and the profile curves have different shapes. In addition, one can see that for FF beams the relative dose decreases as the depth increases, in contrast to FFF where the opposite effect is observed, i.e. the dose increases. It is a result of more low-energy radiation coming from the FF, while for FFF beams the relative dose increases because without the FF, more high-energy photons are present.

Changes of the relative dose in the therapeutic area equal to $80 \%$ of the field size are important for treatment planning in patients whose tumours are located deeply. For this type of location, it is usually necessary to enlarge the field size, which in turn undesirably increases the dose in healthy tissues lying nearby the tumour. Considering the effects

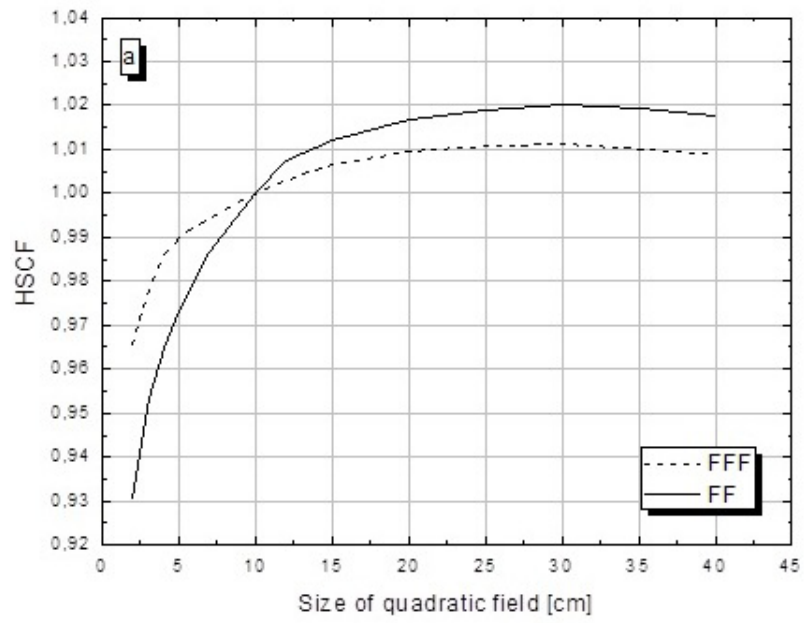

discussed above, it seems that the use of unflattened FFF beams in radiotherapy planning is justified.

\section{Output factors (HSCF, TSCF)}

The curves showing the comparison of HSCF and TSCF output factors for $6-\mathrm{MV}$ and $10-\mathrm{MV}$ FF and FFF beams are presented in Figs. $4 a, 4 b$ and $5 a, 5 b$, respectively. An analysis of the shapes of the obtained curves may be performed by way of adopting two distinct approaches.

The first possibility is to compare the differences between each value of the scattering coefficients for a size of a field greater than $10 \times 10 \mathrm{~cm}^{2}$. That field is a reference field, and the scatter values are normalized to 1 . Fields larger than $10 \times 10 \mathrm{~cm}^{2}$ were taken into account since the scattering is even larger. In addition, if one directly compares the values for two beams, FF and FFF, with the same acceleration voltage, the differences are immediately noticeable.

Nonetheless, the second more appropriate way of analysing the curves of dispersion coefficients depends on a direct comparison of the differences between HSCF and TSCF values for the largest and smallest field sizes for two photon beams $[20,21]$. This shows

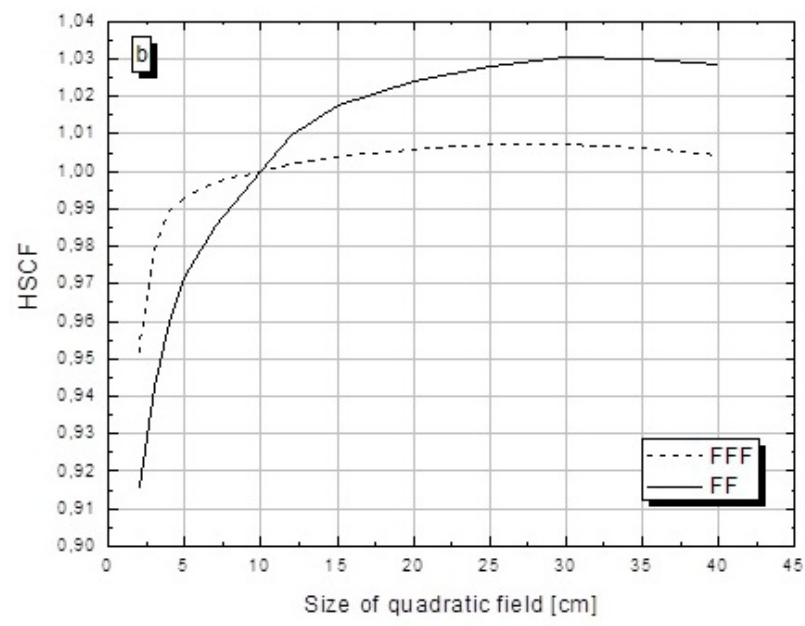

Fig. 4. Head scatter correction factor curves from the side of the irradiation field for flattening filter (FF) and flattening filter-free (FFF) (a) 6-MV and (b) 10-MV photon beams. 

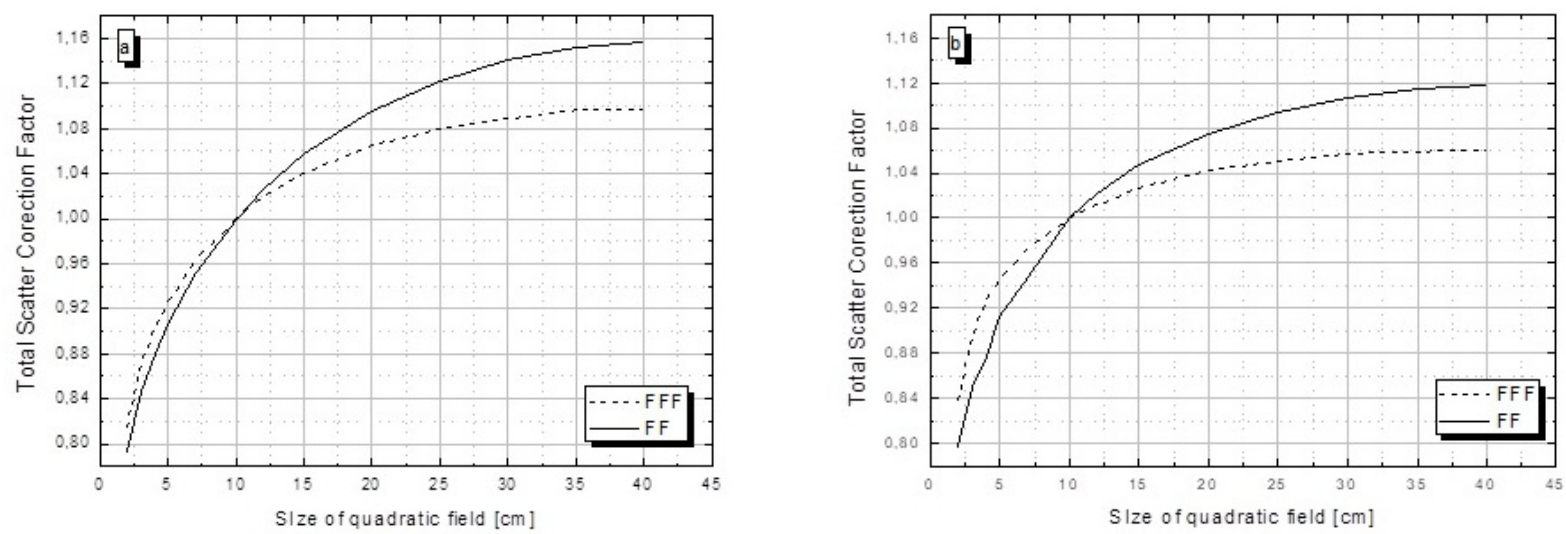

Fig. 5. Total scatter correction factor curves from the side of the irradiation field for flattening filter (FF) and flattening filter-free (FFF) (a) 6-MV and (b) 10-MV photon beams.

the spread of scatter values for the extreme sizes of the measured fields for one type of energy. Moreover, it shows that the dispersion is lower for unflattened beams for the same value of energy.

\section{Head scatter correction factor (HSCF)}

HSCF was measured, when the ionization chamber was placed at a distance of $100 \mathrm{~cm}$ from the radiation source for a range of fields from $2 \times 2 \mathrm{~cm}^{2}$ to $40 \times 40 \mathrm{~cm}^{2}$. The two approaches outlined above may be applied to undertake an analysis of the obtained values shown graphically in Figs. $4 a$ and $4 b$.

Under the first approach, as shown in Fig. 4a, one can see that the FFF beam has smaller HSCF values for all sizes of fields and the differences, as compared to FF, are less than 0.01 . Under the second approach, the difference of HSCF data presented in Fig. $4 \mathrm{a}$ for FF is approximately equal to 0.087 , while for the beams of unflattened FFF 0.044 . Thus, a decrease of $50.6 \%$ was observed. On the whole, both adopted approaches confirm that the HSCF values for unflattened FFF photon beams are substantially smaller than those for FF beams.

A similar situation was observed for higher energy under investigation generated by the voltage of $10 \mathrm{MV}$. According to the first criterion, the maximum difference between the HSCF value for fields larger than $10 \times 10 \mathrm{~cm}^{2}$ is 0.025 . By the second criterion, the HSCF value for 10-MV photon beams for the largest and smallest fields for FF is equal to 0.115 , while for FFF 0.053. The advantage of FFF of the order of $46.1 \%$ was also observed.

\section{Total scatter correction factor (TSCF)}

TSCF measured by ionization chamber was placed in a $100-\mathrm{cm}$ distance from the radiation source to the conditions, SSD $=90 \mathrm{~cm}, d_{\text {meas }}=10 \mathrm{~cm}$, for a range of fields from $2 \times 2 \mathrm{~cm}^{2}$ to $40 \times 40 \mathrm{~cm}^{2}$. The same criteria as in HSCF were adopted for the TSCF analysis for flattened and unflattened beams. The measurement results are shown in Figs. 5a and 5b.

The TSCF curves, as compared to the HSCF curves shown in Figs. $4 \mathrm{a}$ and $4 \mathrm{~b}$, are of a different nature. For the fields larger than $10 \times 10 \mathrm{~cm}^{2}$, the TSCF values increase. Moreover, the differences in the values of TSCF for FF beams in comparison to the values of that coefficient for FFF beams also increase. The maximum value of the difference is 0.061 for the field size of $40 \times 40 \mathrm{~cm}^{2}$.

The difference between the extreme values of the TSCF is significantly bigger. For FF photon beams, it is equal to 0.367, while for FFF 0.281. Although the ratio between the values is less than two (1.310), as was the case with the HSCF, it can be seen that the absolute values of the total scatter correction factor for FFF photon beams are smaller.

Figure $5 \mathrm{~b}$ shows the curves of TSCF changes for the 10 -MV photon beams. In comparison to Fig. $4 \mathrm{~b}$, the ratios of the factors for FF and FFF beams show smaller differences from the maximum value of 0.058 for the field size of $40 \times 40 \mathrm{~cm}^{2}$ to the minimum value of 0.041 for the field size of $2 \times 2 \mathrm{~cm}^{2}$.

The comparison of the HSCF and TSCF results obtained under the second approach produced the same conclusions. The TSCF value between the maximum and minimum field sizes was larger for the higher energy. For flattened beams, the difference is equal to 0.320 , whereas for unflattened beams it is 0.222 .

\section{Physical parameters}

The overall goal of the treatment plan is to fit all the parameters in such a way as to provide the highest probability of the patient's recovery and minimize the treatment time. To this end, treatment plans were prepared by one planner for 10 patients for one irradiated region in the treatment planning system (TPS) MONACO v.3.30, which uses the Monte Carlo algorithm to calculate the dose. In TPS, we based on the same optimization parameters, changed only the type of beam for the same irradiated region. TPSs compared the dose distributions presented in DVHs (called the dose-volume histograms) and the implementation times for the treatment of selected tumours.

The comparison was made for the VMAT technique, which is nowadays a widely used technique of conformal treatment. The advantage of VMAT systems from ensuring that the therapeutic isodose fits precisely the shape of the tumour, while its disadvantage lies the eventuality of irradiation by low doses the relatively large volume of normal tissues. The pre- 

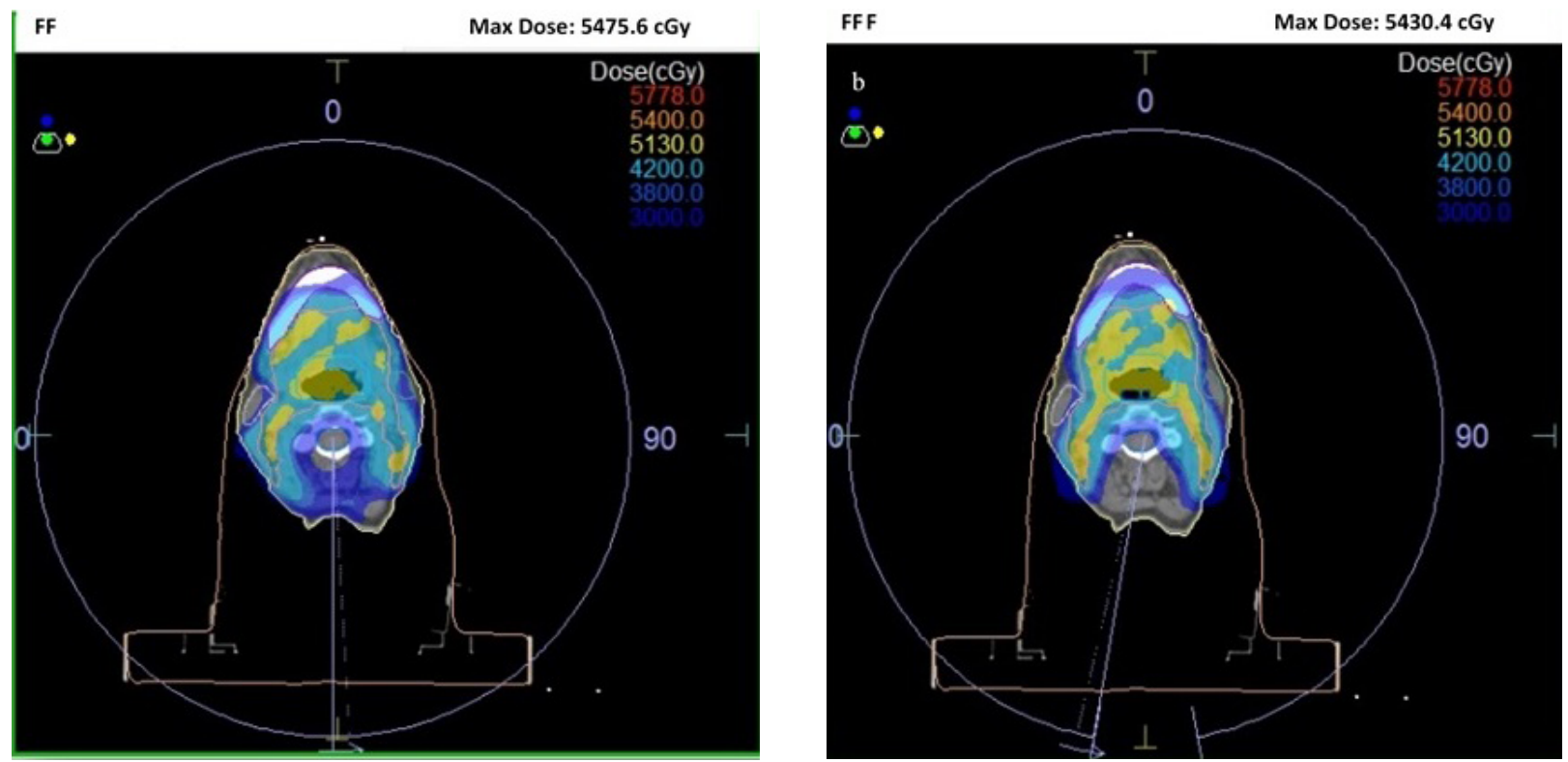

Fig. 6. Dose distributions for the head/neck area using 6-MV photon beams in the treatment planning system (a) with flattening filter (FF) and (b) without flattening filter (FFF) beams.

a)
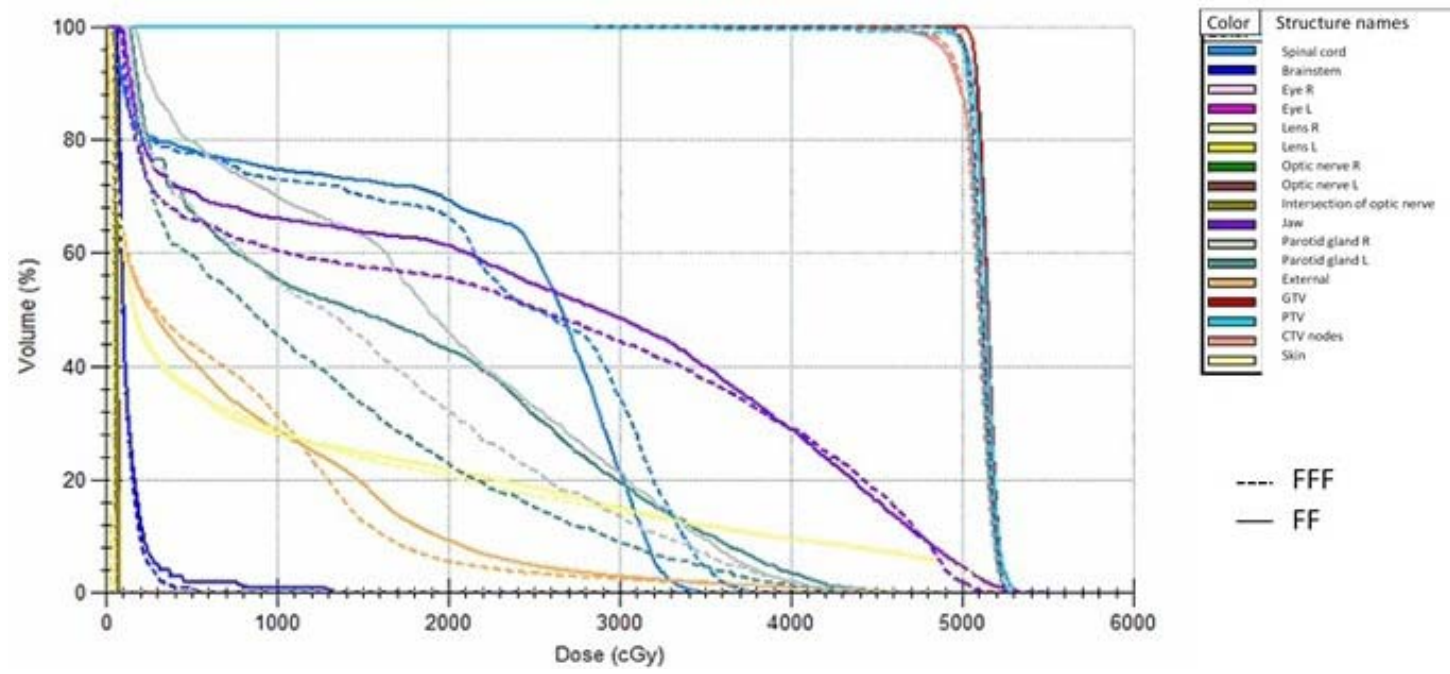

-... FFF

$-\mathrm{FF}$

b)

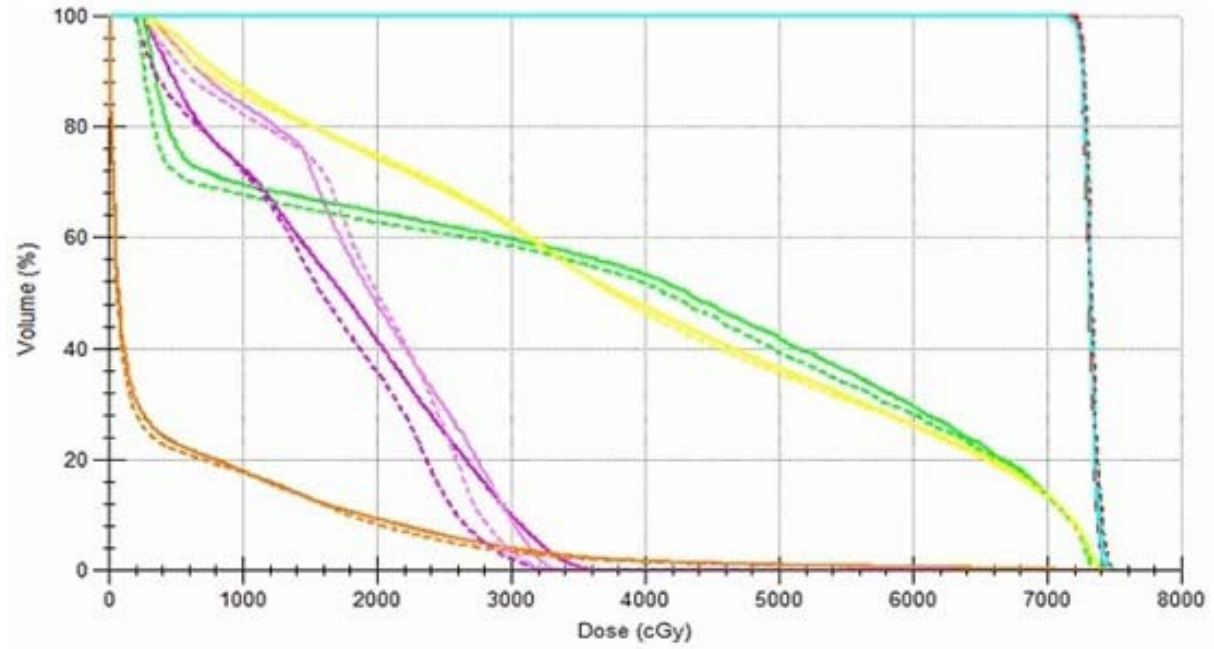

\begin{tabular}{|l|l|l|l|l|l|l}
\hline Color & Structure names \\
\hline
\end{tabular}

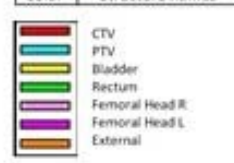

$---\mathrm{FFF}$

$-\mathrm{FF}$

Fig. 7. Dose-volume histograms for (a) the head/neck area and (b) pelvis area using 6-MV photon beams in the treatment planning system with flattening filter (FF) and without flattening filter (FFF) beams. 
Table 5. Comparison of irradiation times for 6-MV FFF beams generated by the Elekta Synergy accelerator and FF and FFF beams generated by the Elekta Versa HD accelerator

\begin{tabular}{lccc}
\hline \multirow{2}{*}{$\begin{array}{l}\text { Tumour region/number of arcs [MU] } \\
\text { Total dose/number of fractions [s] }\end{array}$} & \multicolumn{2}{c}{ Elekta Synergy } & Elekta Versa HD \\
\cline { 2 - 4 } & VMAC technique, fraction dose $<10$ Gy & Agility (FF) & Agility (FFF) \\
\hline Prostate/1 arc & 789 & 762 & 633 \\
60 Gy/30 fr. & 171 & 915 & 1123 \\
Head and neck/2 arcs & 635 & 152 & 182 \\
54 Gy/30 fr. & 293 & 156 & 169 \\
& VMAT technique, fraction dose $>10$ Gy & 1997 & 2710 \\
Lung/1 arc & 2014 & 2281 & 2733 \\
60 Gy/5 fr. & 230 & 245 & 331 \\
Liver/1 arc & 2494 & 130 & 132 \\
60 Gy/5 fr. & 345 & & \\
\hline
\end{tabular}

MU - monitor units.

pared treatment plans used both kinds of photon beams generated with the voltage of 6 MV $[22,23]$.

Figure 6 shows the examples of dose distributions for the head/neck area for FF (Fig. 6a) and FFF (Fig. 6b) photon beams. Using the FFF rather than FF beam, one can see that the tumour is better covered by the isodose $95 \%$ ( $5130 \mathrm{cGy}$ - yellow colour) of the target dose (5400 cGy), while healthy tissues receive lower doses. Moreover, the maximum dose is lower for FFF beam than FF beam in the presented plan of treatment. The clinical applications of FFF beams and the effects of their use throughout the course of treatment were presented on the basis of treatment plans from two regions, head/neck and pelvis, using both types of photon beams. The results of dose-volume histograms are shown in Figs. $7 \mathrm{a}$ and $7 \mathrm{~b}$, respectively.

Analysis of the two histograms reveals that the organs at risk (OAR) receive much lower doses for FFF (dashed lines) than FF (solid lines) beams. This means that the probability of side effects of the therapy connected with the occurrence of secondary cancer tumours is reduced. The curves of the clinical target volume (CTV) and planning target volume (PTV) for FFF beams covered the corresponding curves for FF beams. Thus, the recommendations of ICRU report [24] were fulfilled. These results confirm that the FFF photon beams give a much better dose distribution, in particular for OAR.

Another important parameter affecting the quality and precision of treatment is the implementation time of one fractional dose. The comparison of the treatment time for three types of collimators, MLCi2 for FF beam used in the conventional Elekta Synergy accelerators and Agility for both FF and FFF beams in the accelerator Elekta Versa HD, is provided in Table 5. Table 5 is divided into two parts corresponding to fractional doses lower and higher than $10 \mathrm{~Gy}$, for four different treatment areas: prostate and head/neck and lung and liver, respectively. For each region, the number of arcs, total dose and number of fractions are listed. In all four cases, the time of irradiation is significantly reduced with a simultaneous increase in monitor units (MU) by using photon beams type FFF generated from the accelerator Elekta Versa HD. Hence, the reduction of treatment time increases the patient's comfort during each session and due to patient movements improves the precision of irradiation dose during treatment. Hence, the reduction of treatment time increases the patient's comfort and improves the presicion of irradiation dose during each treatment session.

\section{Discussion}

Five different parameters concerning the quality, usability and performance of FF and FFF beams were studied. Significant differences between these two types of beams were emphasized. The first feature discussed was the PDD curve. The results revealed and confirmed the other authors' conclusion that the shape of the PDD curve is not dependent on the presence of an FF $[1,8]$. The shape of the FFF beam profile would suggest that homogeneity is not maintained in the therapeutic area. However, the current technology allows one to keep the homogeneity of unflattened beams; moreover, some authors $[1,8]$ claim that the definition of individual areas for FFF beams should be changed. This is one of the suggestions for the development of research on FFF beams.

The second parameter under investigation was the stability of the beam and hence of the dose at the edges of the irradiation field in relation to the depth changes. In practice, measurements of the relative doses at the edges of the irradiation field were made. The results presented in Figs. 3a, 3b and described in the literature [2] show that there are apparent differences in dose values of $80 \%$ of the relative field size for FF beams as opposed to FFF beams, where the assigned values are almost identical and do not alter with the depth (Table 1).

Scattering coefficients (output factors) for the FFF beams are significantly smaller than the respective ones for FF beams, meaning that there is less scattered radiation in FFF beams. It can be observed in both graphs which show HSCF (Figs. 4a, 4b) and also TSCF (Figs. 5a, 5b). For both energies, $6 \mathrm{MV}$ and $10 \mathrm{MV}$, it is confirmed that the values of both HSCF and TSCF are definitely smaller for FFF photon beams than the corresponding values 
of those coefficients for conventional beams for the same field size. However, the trend of scattering coefficient growth in line with the increasing field size does not change after removing the FF. The overall decrease in HSCF and TSCF values for unflattened beams is very beneficial for the patient, since in this way the patient is not exposed to an unnecessary additional dose.

All FFF dosimeter parameters are very important, because they have a direct impact on the planning process of the patient's therapy. This research presents tendencies in their behaviour; nonetheless, it should be noted that each medical accelerator may produce slightly different results. For the accelerator Elekta Versa HD, the characteristics of the beams and parameters described in the literature to date were confirmed $[1,2,14]$.

DVHs and the values of the irradiation time (Table 5) show the advantages of FFF photon beams in radiotherapeutic planning and hence benefits for patients. Lower doses to OAR with no changes in the dose delivered to the tumour area and simultaneously shorter irradiation time undermine the future of the conventional FF photon beams in favour of the unflattened FFF [25].

\section{Conclusions}

In summary, one can draw several conclusions that may be relevant to future research:

- There are no observed differences of PDD curves between conventional FF and unflattened FFF photon beams. The presence of an FF does not have any effect on the decrease in the dose in relation to depth.

- There are insignificant differences in the values of relative doses depending on the distance from the beam axis on the edges of the FFF beam profiles for $80 \%$ of the relative field size. For the FF beams, the differences for the same values are significant, which may adversely affect the treatment process.

- The values of TSCF and HSCF coefficients are smaller for FFF beams for the studied field sizes. Thus, the contribution of scattered radiation in the deposited dose is smaller.

- The use of FFF photon beams can improve the quality of patient treatment by:

- reducing the irradiation time of fractional doses by increasing the dose rate [MU/min],

- increasing the accuracy of the deposited dose through the higher stability of patient's positioning due to the shorter irradiation time,

- reducing the undesired dose at OAR and healthy tissues by diminishing the amount of scattered radiation.

The use of radiation FFF beams generated by Elekta Versa HD in the Katowice Oncology Center increases the applicability of techniques such as: VMAT, SRT or SBRT for all locations of cancerous tumours. It is highly probable that the benefits of unflattened beams will lead to their widespread implementation in the radiotherapeutic planning, most probably instead of FF beams. However, there are some specific issues that still have to be addressed. The most important one is the change of the shape of the beam profile, which is directly related to the change in the definition of the therapeutic area and penumbra. Until now, these terms are still not standardized for FFF photon beams.

\section{References}

1. Cashmore, J. (2008). The characterization of unflattened photon beams from a $6 \mathrm{MV}$ linear accelerator. Phys. Med. Biol., 53(7), 1933-1946.

2. Kragl, G., af Wetterstedt, S., Knausl, B., Lind, M., McCavana, P., Knoos, T., McClean, B., \& Georg, D. (2009). Dosimetric characteristics of 6 and $10 \mathrm{MV}$ unflattened photon beams. Radiother. Oncol., 93(1), 141-146.

3. Narayanasamy, G., Saenz, D., Cruz, W., Ha, C. S., Papanikolaou, N., \& Stathakis, S. (2016). Commissioning an Elekta Versa HD linear accelerator. J. Appl. Clin. Med. Phys., 17(1), 179-191.

4. Riis, H. L., Beierholm, A. R., Zimmermann, S. J., Helt-Hansen, J., \& Andersen, C. E. (2014). Measuring output factors for flattening filter free beams: A case study of the Elekta Versa HD. In ESTRO-33, 4-8 April 2014, Vienna, Austria. (EP-1525).

5. Paynter, D., Weston, S. J., Cosgrove, V. P., Evans, J. A., \& Thwaites, D. I. (2014). Beam characteristics of energy-matched flattening filter free beams. Med. Phys., 41, 052103.

6. Mohammed, M., Chakir, E., Boukhal, H., Mroan, S., \& El Bardouni, T. (2016). Evaluation of the dosimetric characteristics of $6 \mathrm{MV}$ flattened and unflattened photon beam. Journal of King Saud University-Science, 29, 371-379.

7. Pichandi, A., Ganesh, K. M., Jerin, A., Balaji, K., \& Kilara, G. (2014). Analysis of physical parameters and determination of inflection point for flattening filter free beams in medical linear accelerator. Reports of Practical Oncology and Radiotherapy, 19, 322-331.

8. Ting, J. (2012). Facts, fiction and future of flattening filter free beams (FFF of FFF beams). In AAPM 54th Annual Meeting, 27 July - 2 August 2012, Melbourne, Australia. Available from https://www.aapm.org/ meetings/2012AM/PRAbs.asp? mid=68\&aid $=19886$.

9. Fogliata, A., Garcia, R., Knoos, T., Nicolini, G., Clivio, A., Vanetti, E., Khamphan, C., \& Cozzi, L. (2012). Definition of parameters for quality assurance of flattening filter free (FFF) photon beams in radiation therapy. Med. Phys., 39(10), 6455-6464.

10. Vassiliev, O. N., Titt, U., Ponisch, F., Kry, S. F., Mohan, R., \& Gillin, M. T. (2006). Dosimetric properties of photon beams from a flattening filter free clinical accelerator. Phys. Med. Biol., 51, 1907-1917.

11. Duane, S. (2013). Dosimetry for flattening filter free (FFF) linac beams and small fields (SF). National Physical Laboratory. Available from dl.icdst.org/pdfs/ files2/58b4dd654592e98362ae5f3e87226fc1.pdf.

12. Kry, S. F. (2013). Flattening filter free accelerators. The University of Texas, MD Anderson Cancer Center, Radiological Physics Center. Available from www.medfys.no/nfmf-documents/Opplastet/downloads/2012/07/Kry_FFF.pdf. 
13. Xiao, Y., Kry, S. F., Popple, R., Yorke, E., Papanikolaou, N., Stathakis, S., Xia, P., Huq, S., Bayouth, J., Galvin, J., \& Yin, F. -F. (2015). Flattening filter-free accelerators: a report from the AAPM Therapy Emerging Technology Assessment Work Group. J. Appl. Clin. Med. Phys., 16(3), 12-29.

14. Budgell, G., Brown, K., Cashmore, J., Duane, S., Frame, J., Hardy, M., Paynter, D., \& Thomas, R. (2016). IPEM Topical Report 1: Guidance on implementing flattening filter free (FFF) radiotherapy. Phys. Med. Biol., 61, 8360-8394.

15. Garcia, R. (2011). Recommendations on QA of FFF beams. Berlin: Varian Oncology Summit.

16. Podgorsak, E. B. (2005). Radiation oncology physics: a handbook for teachers and students. Vienna: International Atomic Energy Agency.

17. Ponisch, F., Titt, U., Vassiliev, O. N., Kry, S. F., \& Mohan, R. (2006). Properties of unflattened photon beams shaped by a multileaf collimator. Med. Phys., 33(6), 1738-1746.

18. Followill, D., Tailor, R., Tello, V., \& Hanson, W. (1998). An empirical relationship for determining photon beam quality in TG-21 from a ratio of percent depth doses. Med. Phys., 25, 1202-1205.

19. Allen, J. (2014). High dose rate mode (flattening filter free) radiotherapy. Clinical advantages of high dose rate mode, available with Agility ${ }^{\mathrm{TM}}$ on Elekta's Versa $H D^{\mathrm{rm}}$ linear accelerator. Crawley: Elekta Ltd.
20. van Gasteren, J. J. M., Heukelom, S., Jager, H. N., Mijnheer, B. J., van der Laarse, R., van Kleffens, H. J., Venselaar, J. L. M., \& Westermann, C. F. (1998). Determination and use of scatter correction factors of megavoltage photon beams. Netherlands Commission on Radiation Dosimetry. (NCRD Report no. 12).

21. Kry, S. F., Vassiliev, O. N., \& Mohan, R. (2010). Outof-field photon dose following removal of the flattening filter from a medical accelerator. Phys. Med. Biol., 55(8), 2155-2166.

22. Cashmore, J. (2013). Operation, characterisation and physical modelling of unflattened medical linear accelerator beams and their application to radiotherapy treatment planning. Unpublished $\mathrm{PhD}$ Thesis, University of Birmingham.

23. Zhu, T. C. (2012). Dosimetric challenge for flattening filter free (FFF) photon beams. In AAPM DVC Spring Symposium, April 20, 2012, Philadelphia, USA. American Association of Physicists in Medicine.

24. ICRU. (1999). ICRU Report 62: Prescribing, recording and reporting photon beam therapy. (Supplement to ICRU Report 50). Journal of the ICRU, 32(1).

25. Georg, D., Knoos, T., \& McClean, B. (2011). Current status and future perspective of flattening filter free photon beams. Med. Phys., 38, 1280-1283. 